Article

\title{
The Measurement Problem from the Perspective of an Information-Theoretic Interpretation of Quantum Mechanics
}

Jeffrey Bub ${ }^{1,2,3}$

${ }^{1}$ Philosophy Department, University of Maryland, College Park 20742, MD, USA;

E-Mail: jbub@umd.edu

${ }^{2}$ Joint Center for Quantum Information and Computer Science, University of Maryland, College Park 20742, MD, USA

${ }^{3}$ Institute for Physical Science and Technology, University of Maryland, College Park 20742, MD, USA

Academic Editor: Olimpia Lombardi

Received: 9 September 2015 / Accepted: 22 October 2015 / Published: 28 October 2015

\begin{abstract}
The aim of this paper is to consider the consequences of an information-theoretic interpretation of quantum mechanics for the measurement problem. The motivating idea of the interpretation is that the relation between quantum mechanics and the structure of information is analogous to the relation between special relativity and the structure of space-time. Insofar as quantum mechanics deals with a class of probabilistic correlations that includes correlations structurally different from classical correlations, the theory is about the structure of information: the possibilities for representing, manipulating, and communicating information in a genuinely indeterministic quantum world in which measurement outcomes are intrinsically random are different than we thought. Part of the measurement problem is deflated as a pseudo-problem on this view, and the theory has the resources to deal with the remaining part, given certain idealizations in the treatment of macrosystems.
\end{abstract}

Keywords: quantum information; measurement problem; information-theoretic interpretation

\section{Introduction}

The salient feature of quantum phenomena that resists any classical explanation is the existence of nonlocal correlations associated with entangled quantum states. Schrödinger ([1], p.555) identified 
entanglement as "the characteristic trait of quantum mechanics, the one that enforces its entire departure from classical lines of thought".

For two quantum systems in a pure entangled state, Colbeck and Renner [2,3] have shown that there can't be a variable $\lambda$, associated with the history of the systems before the preparation of the entangled state in the reference frame of any inertial observer, that provides information about the outcomes of measurements on the systems, so that the marginal probabilities conditional on $\lambda$ for measurement outcomes on the separate systems differ from the quantum probabilities. In other words, the information in a pure entangled state about the probabilities of measurement outcomes is as complete as it could be, which means that the measurement outcomes are intrinsically random events in the sense that they are uncorrelated with any prior events.

Gleason ([4], p. 886) used the term "intertwined" to describe the intricate meshing of commuting and noncommuting quantum observables in a seminal paper in which he proved that it follows from this feature that there is essentially only one way to define probabilities on the structure of quantum properties defined by the two-valued observables in a Hilbert space of three or more dimensions. Intertwinement imposes objective pre-dynamic probabilistic constraints on correlations between events in a similar sense to which Minkowski space-time imposes kinematic constraints on events. The probabilistic constraints of the correlational structure provide the framework for the physics of a genuinely indeterministic universe. They characterize the structure of information for correlations that include the nonlocal correlations of entangled quantum states, which can only occur between intrinsically random events.

An information-theoretic interpretation of quantum mechanics in the sense I have in mind begins with the idea that the relation between quantum mechanics and the structure of information is analogous to the relation between special relativity and the structure of space-time. Insofar as quantum mechanics deals with a class of probabilistic correlations that includes correlations structurally different from correlations that arise in classical theories, the theory is about the structure of information.

Shannon's classical theory of information [5] was about the communication of messages as signals via a communication channel. The fundamental question for Shannon was how to quantify the minimal physical resources required to represent messages produced by a source, so that they could be communicated via a channel and reconstructed by a receiver. For this problem, and related communication problems, the meaning of the message is irrelevant, as Shannon pointed out:

The fundamental problem of communication is that of reproducing at one point either exactly or approximately a message selected at another point. Frequently the messages have meaning; that is they refer to or are correlated according to some system with certain physical or conceptual entities. These semantic aspects of communication are irrelevant to the engineering problem. The significant aspect is that the actual message is one selected from a set of possible messages. The system must be designed to operate for each possible selection, not just the one which will actually be chosen since this is unknown at the time of design.

—Claude E. Shannon ([5], p. 379)

The classical theory of information was about the "engineering problem" of communicating messages over a channel efficiently. In this sense, the concept of information has nothing to with anyone's 
knowledge and everything to do with the stochastic or probabilistic process that generates the messages. What we have discovered is that the 'engineering' possibilities for representing, manipulating, and communicating information in a quantum world are different than we thought, irrespective of what the information is about.

The purpose of this paper is to consider the consequences of this view for the measurement problem of quantum mechanics.

\section{Some Clarifications}

To say that quantum mechanics is about the structure of information is not to endorse a view that "our reality is ultimately made up of information" and that "the laws of Nature are information about information" ([6], pp. 13, 215, 218). The idea that the basic building blocks of reality might be information is intriguing, but I have no idea how to make sense of this. It can't be information in the sense that Shannon had in mind.

Harvey Brown and Chris Timpson [7] have criticized my earlier remarks [8-10] along these lines, particularly with reference to the Clifton-Bub-Halvorson result [11] that the observables and state space of a theory formulated in $C^{*}$-algebraic terms must be quantum mechanical if the theory satisfies certain information-theoretic constraints. Briefly, the critique focuses on my reference to Einstein's distinction between principle and constructive theories, and the claim that quantum mechanics, like special relativity, should be understood as a principle theory, where the principles concern space-time structure in the case of relativity, and information-theoretic structure in the case of quantum mechanics:

Even more significantly for our purposes, at the end of their paper CBH suggested an analogy between their characterization of quantum mechanics and Albert Einstein's special theory of relativity (henceforth SR). The "foundational significance" of the $\mathrm{CBH}$ derivation is, according to these authors, that quantum mechanics should be interpreted as a principle theory, in the sense of the term that Einstein used to describe his 1905 formulation of SR. $\mathrm{CBH}$ saw their constraints as analogous to the principles-the relativity principle and the light postulate - used by Einstein to derive the nature of relativistic kinematics. ... Einstein's principle theory route was based on a policy of despair, and represented a strategic retreat from the more desirable but, in his view, temporarily unavailable constructive approach.

- Harvey Brown and Chris Timpson ([7], p. 3)

The principle versus constructive distinction is a bit of a red herring here, as is Einstein's view about the sense in which a principle theory could provide an understanding of physical processes. Later in the paper, Brown and Timpson say (my italics):

Like the above-mentioned ether theorists [Lorentz, Larmor, and Poincaré], Einstein realized that the covariance of Maxwell's equations - the form invariance of the equations-is achieved when the relevant coordinate transformations take a very special form, but Einstein was unique in his understanding that these transformations, properly understood, encode new predictions as to the behaviour of rigid bodies and clocks in motion. That is why, in Einstein's mind, a new understanding of space and time themselves was in the offing. 
- Harvey Brown and Chris Timpson ([7], p. 6)

Quantum mechanics was born with Heisenberg's seminal 1925 "Umdeutung" paper [12] "On the quantum-theoretical re-interpretation [Umdeutung] of kinematical and mechanical relations". Heisenberg thought that the discrete orbits of Bohr's atomic theory were an artificial theoretical fix that "saved the appearances", but this was not the right way to think about the structural features of atoms responsible for the emission and absorption spectra of gases. His proposal was to re-interpret classical mechanical quantities, like position, momentum, energy, angular momentum, as operations, later represented by operators that act on and transform the states of quantum systems. The aim was to explain the discrete frequencies of light emitted by atoms without appealing to electron orbits, and later to explain other phenomena that couldn't be explained by classical physics. In a 1925 letter to Wolfgang Pauli, Heisenberg wrote :

All of my meagre efforts go toward killing off and suitably replacing the concept of the orbital paths that one cannot observe.

—David C. Cassidy ([13], p. 197)

In the same year, Max Born and Pascual Jordan published the first formulation of quantum mechanics in the first part of a two-part paper "Zur Quantenmechanik" [14]. Part II of this paper, referred to by historians as the "three-man paper", was co-authored with Heisenberg and published a year later [15].

A noncommutive mechanics with the sort of intertwinement of commuting and noncommuting observables characteristic of quantum mechanics noted by Gleason "encodes new predictions as to correlations between measurement outcome events," which is why "a new understanding of the structure of information is in the offing," analogous to the new relativistic understanding of space and time required by the way rigid bodies and clocks in motion behave. This is the information-theoretic interpretation of quantum mechanics in the sense I have in mind.

\section{Consequences for the Measurement Problem}

Itamar Pitowsky distinguishes a "big" measurement problem from a "small" measurement problem [16,17]. The "big" measurement problem is the problem of explaining how the dynamics of a quantum measurement process produces a definite outcome. The "small" measurement problem is the problem of explaining how a classical probability distribution over macroscopic measurement outcomes emerges dynamically in a measurement process.

On the information-theoretic interpretation, the "big" measurement problem is a pseudo-problem. If the universe is genuinely indeterministic and measurement outcomes are intrinsically random, then it isn't possible to provide a dynamical explanation of how a system produces a definite outcome when it's measured-that's what it means for the measurement outcomes to be intrinsically random. The random selection of a definite outcome in a quantum measurement process is a feature of the nonclassical structure of information in a quantum world, just as Lorentz contraction is a feature of the Minkowski structure of space-time in a relativistic world. 
In a classical theory, probabilistic correlations-consider correlations between two systems for simplicity - have the structure of a simplex (a line with two end points or vertices, a triangle with three vertices, a tetrahedron with four vertices, and so on). A point in the interior of the simplex represents an array of joint probabilities for all possible measurements and all possible measurement outcomes on the systems. The vertices of the simplex represent deterministic 0,1 probabilities. A simplex is the only convex polytope for which there is a unique representation of any interior point as a convex combination of the vertices. So classical theories are rather special: there is a unique representation of the associated joint probabilities as probability measures over deterministic states. Correlations that can be simulated with local or classical resources form a convex polytope, the local correlation polytope, which is not in general a simplex. A simulation corresponds to a causal explanation of the correlations in a simplex theory. The local correlation polytope is included in a convex set with a continuous boundary representing quantum correlations, and this convex set is in turn included in the set of correlations that satisfy a "no signaling" constraint, a convex polytope (also not a simplex) with vertices representing Popescu-Rohrlich (PR) boxes [18]. In a classical simplex theory where all quantities are definite, it is possible to give a dynamical account of how a particular measurement outcome is produced in a measurement process: the (pure) state of the system undergoes a deterministic reversible dynamical transition from one vertex of the simplex to a vertex associated with the measurement outcome. For correlations that can't be simulated in a simplex theory, there is no analogous dynamical account (if you're not a Bohmian or an Everettian—see below). Any such account would be inconsistent with the "no signaling" principle.

Here's a way to see this. The state of a quantum system can be specified by the probabilities of the outcomes of measurements on some "informationally complete" or "fiducial" set of observables. For a qubit, the observables $X, Y, Z$ form an informationally complete set. Since these observables don't commute, one would need to measure $X, Y, Z$ on many qubits, all in the same quantum state, to estimate the state from the statistics of the measurement outcomes. Suppose Alice sends Bob a qubit in an unknown quantum state and Bob measures the observable $X$. If there were a deterministic reversible dynamics that described how Bob's measurement outcome came about, Bob could reverse the dynamical evolution to recover the original state after a measurement. Bob could measure the observables $X, Y, Z$ as many times as he liked on the same qubit and identify the unknown state to any arbitrary accuracy from the statistics of the measurement outcomes.

Now suppose that Alice and Bob share an entangled state, say the Bell state $\left|\phi^{+}\right\rangle=\frac{1}{\sqrt{2}}(|0\rangle|0\rangle+$ $|1\rangle|1\rangle)=\frac{1}{\sqrt{2}}(|+\rangle|+\rangle+|-\rangle|-\rangle)$, where $|+\rangle=\frac{1}{\sqrt{2}}(|0\rangle+|1\rangle),|-\rangle=\frac{1}{\sqrt{2}}(|0\rangle-|1\rangle)$. Suppose Alice chooses to measure either the observable $Z$ associated with the basis $|0\rangle,|1\rangle$, or the observable $X$ associated with the basis $|+\rangle,|-\rangle$. If she measures $Z$, her qubit ends up in the state $|0\rangle$ or $|1\rangle$ with equal probability. If she measures $X$, her qubit ends up in the state $|+\rangle$ or $|-\rangle$ with equal probability. The state of Bob's qubit is correlated with the state of Alice's qubit, so after Alice's measurement Bob's qubit will be in an equal weight mixture of the states $|0\rangle,|1\rangle$, or in an equal weight mixture of the states $|+\rangle,|-\rangle$. If Bob could identify the quantum state of the single qubit in his possession, he could distinguish these two mixtures. Alice could signal to Bob, effectively instantaneously, because Alice and Bob could be any distance apart. So a dynamical account of how a definite measurement outcome is selected would be inconsistent with the 'no signaling' principle. It follows that there can't be a deterministic reversible 
dynamics that describes how Bob's measurement outcome is produced in a measurement process. If Bob gains information about an observable in an informationally complete set in a measurement, there must be a corresponding loss of information about other observables in the informationally complete set. The irreducible information loss on measurement is a generic feature of any no-signaling theoretical explanation of correlations that lie outside the local correlation polytope.

A quantum "measurement" is a bit of a misnomer and not really the same sort of thing as the measurement of a property of a classical system, like the temperature of a liquid, or the length of a table top. It involves putting a quantum system like a photon in a situation, say a beamsplitter or an analyzing filter, where the photon has to make an intrinsically random transition to one of two macroscopically distinct alternatives recorded in a device like a photon detector. The quantum probabilities for the values of a polarization observable in a quantum state can't be understood in the classical sense as quantifying ignorance about a particular pre-measurement value of the polarization, or ignorance about an "instruction set" that tells a quantum system what value to produce in a measurement of the polarization. If you could understand quantum probabilities in this way, then you wouldn't get the correlations of entangled states.

So quantum probabilities are understood as probabilities of "what you'll obtain if you measure". This is satisfactory only if a measurement is a process in which probabilities of "what you'll obtain if you measure" become probabilities that can be interpreted in the classical sense as measures of ignorance over several possibilities, one of which actually happens. Otherwise, even after a measurement, you still have probabilities of "what you'll obtain if you measure". What you want is to be able to drop the "if you measure" qualification after a measurement with a macroscopic measuring instrument and recording device, and interpret "what you'll obtain" as "what's there". As Einstein put it ([19], p. 907), you want the moon to be there, and not only there when you look (measure). Is this justified in a quantum universe, and if so, how? This is the "small" measurement problem.

The position of a macroscopic pointer, or Schrödinger's cat being alive or dead [20], is a collective property of an enormous number of microsystems making up the pointer or the cat. A quantum model of a macrosystem treats a macrosystem as composed of a potentially infinite number of microsystems. Macro-observables associated with collective properties can be defined for such an idealized macrosystem, and it turns out that, in the limit, as the number of constituent microsystems goes to infinity, the macro-observables all commute with each other, and with all other observables associated with a finite number of microsystems. For a microsystem, the only observables that commute with all other observables are the zero or null observable and the unit or identity observable, which form the "center" of the noncommutative algebra of observables. For a macrosystem, the center of the algebra contains the null observable and the identity observable, as well as all the macro-observables. Since the macro-observables are in the center of the algebra and commute with all observables, they can be associated with definite values, like the observables of a classical system. Different macrostates correspond to different values of the macro-observables. Technically, the state space (Hilbert space) of a macrosystem breaks up into orthogonal "superselection sectors", and the different values of a macro-observable correspond to these superselection sectors. The existence of superselection sectors is associated with a superselection rule that limits pure state superpositions to states in a particular superselection sector, so there are no superpositions of macrostates. See, for example, [21,22]. 
If a quantum system like a photon is measured by a macroscopic measuring and recording device, you end up with a mixed state, with probabilities over the different possible measurement outcomes (each of which corresponds to a superselection sector) that can be interpreted as classical probabilities in the ignorance sense. In other words, these probabilities are probabilities of "what's there" rather than "what you'll obtain if you measure". The measurement outcomes are definite because the macroscopic measuring and recording device, as far as its macro-observables are concerned, is a classical system, with a commutative algebra of observables, which is equivalent to saying that the algebra of macro-properties is a Boolean algebra. (Geometrically, a Boolean algebra corresponds to a simplex: there is a one-one correspondence between the vertices, edges, and faces of a simplex and the elements of a Boolean algebra. The vertices correspond to Boolean atoms, and the edges and faces correspond to compound Boolean elements.) Putting it in terms of Schrödinger's cat, the cat doesn't end up in an entangled state, where the probabilities defined by the coefficients of the entangled state represent probabilities of "what you'll obtain if you measure" the cat, that is, if you open the chamber and look. Rather, the atom-plus-device-plus-cat ends up in a mixed state in which the cat is either definitely alive or definitely dead, with the appropriate probabilities, interpreted in the usual classical sense as representing ignorance about the two alternatives, one of which actually happens.

This is not to deny that a macroscopic system like a cat could, in principle, exhibit quantum effects of interference or entanglement if it's the object of a quantum measurement with some other system as the measuring instrument, as in recent experiments designed to show that relatively large systems like optomechanical oscillators can be in quantum superpositions or entangled states [23]. In Schrödinger's thought experiment, the cat is treated as the ultimate measuring instrument that registers the decay of the radioactive atom. On the information-theoretic interpretation, the system that plays this role, in Schrödinger's experiment or in an interference experiment with an optomechanical oscillator, must be capable of being modeled as a system with commuting macro-observables, so the macro-properties form a Boolean algebra.

Here's a rough outline of how this works in an idealized "toy model” by Klaus Hepp [24]. In Hepp's model, a macroscopic measurement "pointer" is represented as an infinite array of qubits. The states of the infinite array in which all but a finite number of qubits are in the state $|0\rangle$ belong to one superselection sector, and the states of the infinite array in which all but a finite number of qubits are in the state $|1\rangle$ belong to a second superselection sector. These two superselection sectors represent two macrostates of the pointer, associated with the pointer readings 0 and 1 . Initially, the infinite array representing the macroscopic pointer is in the state with all qubits in the state $|0\rangle$, so the pointer reads 0 . The measured system is a single qubit, $Q$. If $Q$ is in the state $|0\rangle$, the array qubit states are unchanged, but if $Q$ is in the state $|1\rangle$, the measurement interaction flips the array qubit states from $|0\rangle$ to $|1\rangle$ in order, as time goes on. If $Q$ is in a superposition of the states $|0\rangle$ and $|1\rangle$, you end up after the measurement interaction with a mixed state over the two pointer macrostates, in which the 0 pointer reading state is correlated with the state $|0\rangle$ of $Q$, and the 1 pointer reading state is correlated with the state $|1\rangle$ of $Q$. That's what you want: at the end of the measurement, the measured system $Q$ and the measuring instrument end up in a mixed state, which can be interpreted as a classical probability distribution over definite measurement outcomes. The probabilities of the initial state of $Q$, probabilities of "what you'll obtain if you measure", become classical probabilities in the ignorance sense over the two possible measurement outcomes, the 
definite pointer reading states 0 and 1 , which are correlated with the states $|0\rangle$ and $|1\rangle$ of $Q$ (the states corresponding to the two possible values of the measured observable).

Now, as Bell pointed out in a critique of Hepp's model [25], this only occurs at the infinite limit, and in Hepp's model the idealization involves not only considering the limit for an infinite number of qubits, but also the infinite time limit—at any finite time after the measurement interaction begins, only a finite number of qubits in the array are flipped, and so an initial pure state in a superselection sector remains in that superselection sector.

What do these infinite limits mean physically, in particular the infinite time limit? Landsman points out ([21], p. 514) that a measurement is comparable to a scattering process and, for measurement as for decoherence, although the process approaches the behavior at the limit very rapidly, there's no finite time at which the process is over:

Indeed, what would it mean for scattering to be over after some finite time? Which time? As we shall see ... the theory of decoherence requires the limit $t \rightarrow \infty$ as well, and largely for the same mathematical reasons. There as well as in Hepp's approach, the limiting behavior actually tends to be approached very quickly (on the pertinent time scale), and one needs to let $t \rightarrow \infty$ merely to make terms $\sim \exp ^{-\gamma t}$ (with $\gamma>0$ ) zero rather than just very small.

—Nicolaas P. Landsman ([21], p. 514)

Similar idealizations are typical in classical statistical physics. A macroscopic classical system has "emergent" properties that are not properties of its constituent microsystems. For example, a liquid can freeze to a solid when cooled and evaporate to a gas when heated, but the solid-liquid-gas phases of a substance are not well-defined properties of individual molecules or finite collections of molecules, and neither is the temperature. Being a solid or a liquid or a gas, or having a certain temperature, is a collective property of enormous numbers of molecules. In classical physics, collective properties like this, which are insensitive to adding or removing any finite number of molecules, are described theoretically in terms of macro-observables associated with potentially infinite numbers of molecules, and the theoretical analysis necessarily involves idealizations. A glass of water has roughly $10^{24}$ molecules, but classical statistical mechanics produces a phase transition from a liquid to a gas, or a liquid to a solid, only if the system is idealized as an infinite number of molecules. The phase transition occurs at the infinite limit, but not for any finite number of molecules.

\section{Alternatives}

The standard alternatives to this information-theoretic interpretation are Bohm's theory [26,27] or the Everett interpretation [28,29]. Both treat indefiniteness at the quantum level and the intrinsic randomness of quantum events as only apparent, but the devil is in the details.

In Bohm's theory, the values of hidden variables provide missing information that resolves the indefiniteness at the microlevel and, together with the dynamics, explains how measurement outcomes come about, and the correlations between measurement outcomes at the macrolevel. That's the general idea, but the picture is not as straightforward as it might seem at first sight. There are cases where events are registered as occurring when the particle trajectories in Bohm's theory, which are supposed to explain 
the occurrence of these events, are located somewhere else [30-32]. See, for example, the critique by Reinhard Werner that Bohm's theory doesn't really do what it's claimed to do [33].

The Everett interpretation is fundamentally a proposal to solve the "big" measurement problem: a measurement produces a definite outcome in each of many worlds that emerge through the dynamics of decoherence. The basic idea is that the universal quantum wave function is all there is, and each component or "branch" of the wave function evolves separately and represents a complete world as we experience it. Everettians see this as really just standard quantum mechanics, not one interpretation among rivals, but there are some problems and loose ends that complicate the Everettian picture.

The representation of a wave function as a specific superposition of components or branches depends on the basis in the quantum state space, the "coordinate system" with respect to which the wave is represented as superposition. Something physical should determine the basis, since the worlds associated with the branches of the universal wave function depend on the basis. The modern version of the Everett interpretation $[34,35]$ proposes that the basis is selected by decoherence, a feature of the interaction between a macrosystem and its environment. The picture is that a multiplicity of worlds "emerges" as a feature of the universal wave function through decoherence as a dynamical process, which occurs spontaneously with respect to a position basis. In this "multiverse", we ourselves are split into different versions with different experiences associated with the worlds on the different branches of the universal wave function. In particular, the experiences of different outcomes of a quantum measurement occur in worlds on different branches.

The idea is ingenious but the full story is a lot more complicated than this summary suggests. What you get from decoherence is a basis of approximately localized positions, and the worlds that "emerge" in this dynamical process are only well-defined in an idealized sense. You can't, for example, specify how many worlds there are of a certain sort, even in principle, or precisely when worlds are formed. Everettians argue that, properly understood, this is not a problem, but the detailed clarification is complex and controversial.

There's also a problem in making sense of probability in a multiverse, where everything that can happen does happen. The contemporary way of resolving this problem is to argue that there's a sense in which a rational agent in a world can be uncertain about which branch the agent will end up in after the universal wave function undergoes further branching as the result of a measurement. Such an agent can have rational degrees of belief about the outcomes of quantum measurements, even though all possible outcomes occur on different branches and the agent is split between the branches. Given certain assumptions about what it means to be rational in the face of uncertainty, an agent's degrees of belief will agree with the Born probabilities. The decoherence solution to the basis problem, and the analysis of probability and the derivation of the Born rule, are problematic and the subject of a continuing debate between Everettians and their opponents. See, in particular, the critique by Adrian Kent [36].

\section{Conclusion}

So where does this leave things? In a genuinely indeterministic quantum universe described by a noncommutative mechanics, the quantum probabilities of "what you'll obtain if you measure", which arise as features of the noncommutative structure at the microlevel, must ultimately be related to 
probabilities of "what's there" in the macroworld in the classical ignorance sense. That's the "small" measurement problem. This works out if the macrosystems used to probe the microworld are modeled as infinite quantum systems, which is how a physical explanation deals with macroscopic descriptions that are insensitive to certain variations at the microlevel.

Critics will object with Bell [37] that this involves FAPP ("for all practical purposes") reasoning, which makes quantum mechanics a predictive instrument rather than a fundamental theory of matter, of interest only to "shopkeepers and engineers", as Einstein put it:

If one wants to consider the quantum theory as final (in principle), then one must believe that a more complete description would be useless because there would be no laws for it. If that were so then physics could only claim the interest of shopkeepers and engineers; the whole thing would be a wretched bungle.

—Albert Einstein ([38], p. 39)

The conclusion here is rather different. The transition from "what you'll obtain if you measure" to "what's there" in a quantum measurement process, where "what's there" is intrinsically random, is like a phase transition. Quantum mechanics can model what happens, but certain idealizations in the mathematical description of the macrosystems used to probe the quantum world are unavoidable. This was Bohr's position, that a coherent quantum physics requires that observers and the recording infrastructure should be left out of the quantum theoretical story and described classically:

It is decisive to recognize that, however far the phenomena transcend the scope of classical physical explanation, the account of all evidence must be expressed in classical terms. The argument is simply that by the word "experiment" we refer to a situation where we can tell others what we have done and what we have learned and that, therefore, the account of the experimental arrangement and the results of the observations must be expressed in unambiguous language with suitable application of the terminology of classical physics.

—Niels Bohr ([39], p. 209)

Heisenberg added the idea of a movable "cut" between the part of the world doing the measuring and the part being measured. The cut is movable because any part of the world can, in principle, be described purely quantum mechanically, but the idea is that some part of the world-the ultimate measuring and recording device in any application of the theory-must be left out of the quantum mechanical description and described classically.

Bohr's comment is hardly an argument. On the information-theoretic interpretation, the requirement that the experimental arrangement and observational outcomes should be given a "classical" account is understood as the constraint that the ultimate measuring instrument in any quantum experiment should be modeled as a commutative or Boolean system.

To sum up: The "big" measurement problem disappears with the recognition that quantum mechanics is about the structure of information in a universe in which measurement outcomes are intrinsically random events, so there can't be a dynamical account of how a measurement outcome event, indefinite 
before a measurement, becomes definite. Quantum states, unlike classical states, don't characterize a system in terms of its properties, but rather specify the probabilities of "what you'll obtain if you measure", for example the probabilities that a photon, in a certain state of linear polarization, directed to pass through a beamsplitter, will emerge in the horizontal or vertical channel. The remaining problem is to show how probabilities of "what you'll obtain if you measure" become probabilities of "what's there". This turns out to be a consequence of treating the ultimate measuring instrument in any experimental arrangement designed to probe the quantum world as a commutative or Boolean system. Certain idealizations in the quantum mechanical analysis are unavoidable, but these are analogous to idealizations in the classical treatment of phase transitions.

\section{Acknowledgments}

This article is based on the last chapter of my book Bananaworld: Quantum Mechanics for Primates; Oxford University Press, Oxford, UK, February, 2016.

\section{Conflicts of Interest}

The author declares no conflict of interest.

\section{References and Notes}

1. Schrödinger, E. Discussion of probability relations between separated systems. Proc. Camb. Phil. Soci. 1935, 31, 555-563.

2. Colbeck, R.; Renner, R. No extension of quantum theory can have improved predictive power. Nature Comm. 2011, 2, doi:10.1038/ncomms1416.

3. Colbeck, R.; Renner, R. Is a system's wave function in one-to-one correspondence with its elements of reality? Phys. Rev. Lett. 2012, 108, 150402.

4. Gleason, A.N. Measures on the closed subspaces of Hilbert space. J. Math. Mech. 1957, 6, 885-893.

5. Shannon, C. A mathematical theory of communication. Bell Syst. Tech. J. 1948, 379-423, 623-656.

6. Vedral, V. Decoding Reality; Oxford University Press: Oxford, UK, 2010.

7. Brown, H.R.; Timpson, C.G. Why special relativity should not be a template for a fundamental reformulation of quantum mechanics. In Physical Theory and its Interpretation; Demopoulos, W., Pitowsky, I., Eds.; Springer: New York, NY, USA, 2006; pp. 29-42.

8. Bub, J. Quantum mechanics as a principle theory. Stud. Hist. Phil. Mod. Phys. 2000, 31B, 75-94.

9. Bub, J. Why the quantum? Stud. Hist. Phil. Mod. Phys. 2004, 35B, 241-266.

10. Bub, J. Quantum theory is about quantum information. Found. Phys. 2005, 35, 541-560.

11. Clifton, R.; Bub, J.; Halvorson, H. Characterizing quantum theory in terms of information-theoretic constraints. Found. Phys. 2003, 33, 1561-1591.

12. Heisenberg, W. Über Quantentheoretischer Umdeutung kinematischer und mechanischer Beziehungen. Zeit. f. Phys. 1925, 33, 879-893. 
13. Cassidy, D.C. Uncertainty: The Life and Science of Werner Heienberg; W.H. Freeman: New York, NY, USA, 1992.

14. Born, M.; Jordan, P. Zur Quantenmechanik. Zeit. f. Phys. 1925, 34, 858-888.

15. Born, M.; Heisenberg, H.; Jordan, P. Zur Quantenmechanik II. Zeit. f. Phys. 1926, 35, 557-615.

16. Pitowsky, I. Quantum mechanics as a theory of probability. In Physical Theory and its Interpretation; Demopoulos, W., Pitowsky, I., Eds.; Springer: New York, NY, USA, 2006; pp. 213-240.

17. Bub, J.; Pitowsky, I. Two dogmas about quantum mechanics. In Many Worlds? Everett, Quantum Theory and Reality; Saunders, S., Barrett, J., Kent, A., Wallace, D., Eds.; Oxford University Press: Oxford, UK, 2010.

18. Popescu, S.; Rohrlich, D. Quantum nonlocality as an axiom. Found. Phys. 1994, 24, 379-385.

19. Pais, A. Einstein and the quantum theory. Rev. Mod. Phys. 1979, 51, 863-914.

20. Schrödinger, E. Die Gegenwärtige Situation in der Quantenmechanik. Die Naturwissenschaften 1935, 23, 807-812, 823-828, 844-849.

21. Landsman, N.P. Between classical and quantum. In Handbook of the Philosophy of Science; Butterfield, J., Earman, J., Eds.; North-Holland: Amsterdam, The Netherlands, 2007; Volume 2, Philosophy of Physics; pp. 417-553.

22. Landsman, N.P. When champions meet: Rethinking the Bohr-Einstein debate. Stud. Hist. Phil. Mod. Phys. 2006, 37, 212-241.

23. Carlisle, A.; Kwon, H.; Jeong, H.; Ferraro, A.; Paternostro, M. Limitations of a measurement-assisted optomechanical route to quantum macroscopicity of superposition states. Phys. Rev. A 2015, 92, 022123.

24. Hepp, K. Quantum theory of measurement and macroscopic observables. Helv. Phys. Acta 1972, 45, 237-248.

25. Bell, J.S. On wave packet reduction in the Coleman-Hepp model. Helv. Phys. Acta 1975, 48, 93-98.

26. Bohm, D. A Suggested Interpretation of the Quantum Theory in Terms of "Hidden" Variables, I. Phys. Rev. 1952, 85, 166-179.

27. Bohm, D. A Suggested Interpretation of the Quantum Theory in Terms of "Hidden" Variables, II. Phys. Rev. 1952, 85, 180-193.

28. Everett, H. Relative State Formulation of Quantum Mechanics. Rev. Mod. Phys. 1957, 29, 454-462.

29. Everett, H. The Theory of the Universal Wave Function. In The Many-Worlds Interpretation of Quantum Mechanics; De Witt, B., Graham, N., Eds.; Princeton University Press: Princeton, NJ, USA, 1973.

30. Englert, B.-G.; Scully, M.O.; Süsmann, G.; Walther, H. Surrealistic Bohm trajectories. Zeit. f. Nat. A 1992, 47, 1175-1186.

31. Scully, M.O.; Englert, B.G.; Walther, H. Do Bohm trajectories always provide a trustworthy physical picture of particle motion? Physica Scripta 1998, 76, 41-46.

32. Dürr, D.; Fusseder, W.; Goldstein, S.; Zanghi, N. Comment on "Surrealistic Bohm Trajectories". Zeit.f. Nat. 1993, 48a, 1261-1262. 
33. Werner, R. Guest post on Bohmian Mechanics. Available online: https://tjoresearchnotes. wordpress.com/2013/05/13/guest-post-on-bohmian-mechanics-by-reinhard-f-werner (accessed on 22 October 2015).

34. Wallace, D. The Emergent Multiverse: Quantum Theory According to the Everett Interpretation; Oxford University Press: Oxford, UK, 2012.

35. Wallace, D. Many Worlds? Everett, Quantum Theory, and Reality; Saunders, S., Barrett, J., Kent, A., Wallace, D., Eds.; Oxford University Press: Oxford, UK, 2010.

36. Kent, A. One world versus many: The inadequacy of Everettian accounts of evolution, probability, and scientific confirmation. In Many Worlds? Everett, Quantum Theory, and Reality; Saunders, S., Barrett, J., Kent, A., Wallace, D., Eds.; Oxford University Press: Oxford, UK, 2010; pp. 307-354.

37. Bell, J.S. Against “measurement”. Phys. World 1990, 3, 33-40.

38. Einstein, A. A letter to Schrödinger dated December 22, 1950. In Letters on Wave Mechanics; Przibram, K., Ed.; Philosophical Library, New York, NY, USA, 1967.

39. Bohr, N. Discussions with Einstein on epistemological problems in modern physics. In Albert Einstein: Philosopher-Scientist; Schilpp, P.A., Ed.; The Library of Living Philosophers: Evanston, IL, USA, 1949; Volume 7, pp. 201-241.

(c) 2015 by the author; licensee MDPI, Basel, Switzerland. This article is an open access article distributed under the terms and conditions of the Creative Commons Attribution license (http://creativecommons.org/licenses/by/4.0/). 